\title{
INTEGRABLE POTENTIALS AND HALF-LINE SPECTRA ${ }^{1}$
}

\author{
C. R. PUTNAM
}

1. In the differential equation

$$
x^{\prime \prime}+(\lambda-f) x=0,
$$

let $f=f(t)$ be a real-valued, continuous function on $0 \leqq t<\infty$ and suppose that $\lambda$ is a real parameter. If (1) is of the limit-point type, then (1) and a boundary condition of the type

$$
x(0) \cos \alpha+x^{\prime}(0) \sin \alpha=0, \quad 0 \leqq \alpha<\pi,
$$

determine, for every fixed $\alpha$, a boundary value problem on $0 \leqq t<\infty$ with a spectrum (of $\lambda$-values) $S=S_{\alpha}$ [7]. It is known that the set $S^{\prime}$ consisting of the set of cluster points of $S_{\alpha}$ is independent of $\alpha$; loc. cit. p. 251. The following theorem will be proved:

$\left(^{*}\right)$ If $f(t)$ denotes a real-valued, continuous function on the half-line $0 \leqq t<\infty$ satisfying the condition

$$
\int_{0}^{\infty} f(t) d t \text { converges } \quad\left(\int_{0}^{\infty}=\lim _{T \rightarrow \infty} \int_{0}^{T}\right)
$$

then (1) is of the limit-point type and

$$
S^{\prime}=[0, \infty) \text {. }
$$

It is noteworthy that (3) may exist only conditionally and that

$$
\int_{0}^{\infty}|f(t)| d t<\infty
$$

is not assumed. Actually, if $\left(3^{\prime}\right)$ holds, much more is known. In fact, in this case, there exist asymptotic formulas for the solutions of (1) when $\lambda>0$ ( $[8$, p. 421]; cf. also [7, p. 258], in case $f(t) \rightarrow 0$ as $t \rightarrow \infty$ ) which guarantee, in particular, that $0 \leqq \lambda<\infty$ is in the continuous spectrum for every boundary value problem determined by (1) and (2). Obviously, the requirement (3) is compatible with $T^{-1} \int_{0}^{T}|f(t)| d t$ $\rightarrow \infty$, as $T \rightarrow \infty$, and, in fact, even with the requirement that $\int_{0}^{T}|f(t)| d t \rightarrow \infty$ arbitrarily fast. Thus, if $\phi(t)$ denotes any positive function satisfying $\phi(t) \rightarrow \infty$ as $t \rightarrow \infty$, there exists a continuous function $f(t)$ on $0 \leqq t<\infty$ satisfying (3) and $\phi(T)=o\left(\int\left|f_{0}^{T}(t)\right| d t\right)$, as $T \rightarrow \infty$.

Received by the editors March 15, 1954 and, in revised form, August 6, 1954.

1 This work was supported in part by the National Science Foundation research grant NSF-G481. 
On the other hand, most of the criteria for (4) or $S^{\prime} \supset[0, \infty)$ involve $|f(t)|$ rather than $f(t)$, and, as a consequence, require that $f(t)$ be close to zero "on the average." For instance, it is known that

$$
T^{-1} \int_{0}^{T}|f(t)| d t \rightarrow 0, \quad T \rightarrow \infty,
$$

is enough to guarantee that $S^{\prime} \supset[0, \infty)$, although

$$
\lim \sup T^{-1} \int_{0}^{T}|f(t)| d t<\infty
$$

is not; cf. [3, p. 80]. Moreover, (5) is compatible with $S^{\prime}=(-\infty, \infty)$; cf. [3].

2. Proof of $\left(^{*}\right)$. Since $f$ satisfies (3), it is clear that $\int_{0}^{T}(\lambda-f(t)) d t \rightarrow \infty$ as $T \rightarrow \infty$ whenever $\lambda>0$. It follows that (1) is oscillatory (i.e., every nontrivial solution possesses an infinity of zeros clustering at $+\infty$ ) whenever $\lambda>0$; [10], cf. also [4]. Next, it will be shown that, in view of (3), the equation (1) is nonoscillatory whenever $\lambda<0$. (It is of interest to note here that there are known necessary and sufficient conditions in order that an equation (1) be oscillatory; cf., e.g., [5; 9]. In the present case it will be convenient for later use to give the direct argument below.)

Suppose first that $\lambda$ is arbitrary and that (1) possesses an oscillatory solution $x=x(t)(\not \equiv 0)$ with zeros tending to infinity. If $S<T$ denote two zeros of $x(t)$, a multiplication of (1) by $x$ followed by an integration leads to

$$
\int_{S}^{T} x^{\prime 2} d t=\lambda \int_{S}^{T} x^{2} d t-\int_{S}^{T} f x^{2} d t
$$

An integration by parts of the second integral on the right side of the equation (6) yields

$$
\int_{S}^{T} f x^{2} d t=-2 \int_{S}^{T} x x^{\prime} F(t) d t, \quad F(t)=\int_{0}^{t} f(s) d s .
$$

In view of (3), $F(t)=$ const. $+o(1)$ as $t \rightarrow \infty$, and an application of the Schwarz inequality to the second integral of (7) now implies

$$
\int_{S}^{T} x^{\prime 2} d t=\lambda \int_{S}^{T} x^{2} d t+o\left(\int_{S}^{T} x^{2} d t \int_{S}^{T} x^{\prime 2} d t\right)^{1 / 2},
$$

and hence,

$$
A=\lambda+o\left(A^{1 / 2}\right), \text { where } A=\int_{S}^{T} x^{\prime 2} d t / \int_{S}^{T} x^{2} d t
$$


where the "o term" refers to $S \rightarrow \infty$. It readily follows from (8) that $\lambda \geqq 0$ and so (1) must be nonoscillatory whenever $\lambda<0$.

It follows from the last result that (1) is of the limit-point type and that, in addition, $S^{\prime} \subset[0, \infty) ;[1]$, cf. also [2]. There remains to be shown that the half-line $\lambda \geqq 0$ belongs to $S^{\prime}$. To this end, consider any boundary condition (2) for a fixed value $\alpha$ and let

$$
m_{\alpha}(\lambda)=\min |\lambda-\mu|,
$$

when $\mu$ is in the (closed) set $S_{\alpha}$. It will be shown that

$$
m_{\alpha}(\lambda) \equiv 0 \quad \text { for } \lambda>0 \text { (hence for } \lambda \geqq 0 \text { ), }
$$

and so (4) will follow.

Let $g=g(t)$ denote any function of class $C^{2}$ on the finite interval $0 \leqq t \leqq T$ and satisfying the boundary conditions (2) and

$$
g(T)=g^{\prime}(T)=0 .
$$

Then the argument of $[6$, pp. 579-580] shows that

$$
m_{\alpha}^{2}(\lambda) \int_{0}^{T} g^{2} d t \leqq \int_{0}^{T}(L(g)+\lambda g)^{2} d t \quad\left(L(x) \equiv x^{\prime \prime}-f x\right) .
$$

Next, let $\mu$ and $\epsilon$ be positive and suppose that $g(t)=y(t) h(t)$, where $h(t)=\cos \left(\mu^{1 / 2} t\right)$ and $y(t)$ is a nontrivial (oscillatory) solution of (1) for $\lambda=\epsilon$, so that $L(y)+\epsilon y=0$, and satisfying (2) for $x=y$. Next, let $T$ be chosen so that

$$
y(T)=0 .
$$

In addition, since (1) is of the limit-point type, the number $\epsilon$ can be chosen arbitrarily small and so that the function $y$ satisfies

$$
\int_{0}^{\infty} y^{2} d t=\infty
$$

cf. [7]. It will be supposed that $\mu=\mu(T)$ is chosen so that

$$
\cos \left(\mu^{1 / 2} T\right)=0 ;
$$

hence, as a consequence of (12) and the relation $g^{\prime}=y^{\prime} h+y h^{\prime}, g(t)$ also satisfies (10). In view of

$$
L(g)+\lambda g=(\lambda-\mu-\epsilon) h y+2 y^{\prime} h^{\prime},
$$

the relation (11) and the inequality $(a+b)^{2} \leqq 2\left(a^{2}+b^{2}\right)$ now yield (16) $m_{\alpha}^{2}(\lambda) \int_{0}^{T} h^{2} y^{2} d t \leqq$ const. $\int_{0}^{T}\left[\mu y^{\prime 2}+(\lambda-\mu-\epsilon)^{2} y^{2}\right] d t$.

Next, let $T=T_{1}<T_{2}<\cdots$ denote the positive zeros of $y=y(t)$ 
and choose $\mu_{n}=\mu\left(T_{n}\right)$ (hence $h=h_{n}$ ) so that (14) holds for $T=T_{n}$ and $\mu_{n} \rightarrow \lambda(>0)$. (That this can be done is clear.) It follows from (16) that, as $n \rightarrow \infty$,

$$
m_{\alpha}^{2}(\lambda) \leqq \text { const. } \lim \sup \left[\int_{0}^{T_{n}}\left(\epsilon^{2} y^{2}+\lambda y^{\prime 2}\right) d t / \int_{0}^{T_{n}} h_{n}^{2} y^{2} d t\right] .
$$

A calculation like that of $[6$, p. 581$]$, together with (12), yields

$$
\int_{0}^{T_{n}} h_{n}^{2} y^{2} d t \geqq \frac{1}{2} \int_{0}^{T_{n}} y^{2} d t-\frac{1}{2} \mu_{n}^{-1 / 2}\left(\int_{0}^{T_{n}} y^{\prime^{2}} d t \int_{0}^{T_{n}} y^{2} d t\right)^{1 / 2}
$$

If use is made of (13), a calculation similar to that used in obtaining (8) shows that $A=\epsilon+o\left(A^{1 / 2}\right)$, as $T_{n} \rightarrow \infty$, where

$$
A=A_{n}=\int_{0}^{T_{n}} y^{\prime 2} d t / \int_{0}^{T_{n}} y^{2} d t
$$

This implies however that $A\left(T_{n}\right)<$ const. $\epsilon$ for $T_{n}$ large, and hence, by (18), $\int_{0}^{T_{n}} h_{n}^{2} y^{2} d t \geqq$ const. $\int_{0}^{T_{n}} y^{2} d t>0$ for $T_{n}$ large and for a sufficiently small $\epsilon$. Finally, relation (17) now implies $m_{\alpha}^{2}(\lambda) \leqq$ const. $\left(\epsilon^{2}+\epsilon \lambda\right)$. Since $\epsilon>0$ can be chosen arbitrarily small, relation (9) follows and the proof of $\left({ }^{*}\right)$ is complete.

\section{REFERENCES}

1. P. Hartman, Differential equations with non-oscillatory eigenfunctions, Duke Math. J. vol. 15 (1948) pp. 697-709.

2. P. Hartman and C. R. Putnam, The least cluster point of the spectrum of boundary value problems, Amer. J. Math. vol. 70 (1948) pp. 849-855.

3. P. Hartman and A. Wintner, On perturbations of the continuous spectrum of the harmonic oscillator, Amer. J. Math. vol. 74 (1952) pp. 79-85.

4. W. Leighton, The detection of the oscillation of solutions of a second order linear differential equation, Duke Math. J. vol. 17 (1950) pp. 57-62.

5. C. R. Putnam, An oscillation criterion involving a minimum principle, Duke Math. J. vol. 16 (1949) pp. 633-636.

6. - On the unboundedness of the essential spectrum, Amer. J. Math. vol. 74 (1952) pp. 578-586.

7. H. Weyl, Ueber gewöhnliche Differentialgleichungen mit Singularitäten und die zugehörigen Entwicklungen willkïlicher Funktionen, Math. Ann. vol. 68 (1910) pp. 222-269.

8. A. Wintner, Small perturbations, Amer. J. Math. vol. 67 (1945) pp. 417-430.

9. - A norm criterion for non-oscillatory differential equations, Quarterly of Applied Mathematics vol. 6 (1948) pp. 183-185.

10.- A criterion of oscillatory stability, Quarterly of Applied Mathematics vol. 7 (1949) pp. 115-117.

\section{Purdue University}

